\title{
Long-term Variations in Solar Activity: Predictions for Amplitude and North-South Asymmetry of Solar Cycle 25
}

\section{J. Javaraiah॰•

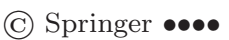

\begin{abstract}
In most of the solar cycles, activity in the northern and southern hemispheres peaks at different times. One hemisphere peaks well before the other and at least one of the hemispheric maxima frequently does not coincide with the whole sphere maximum. Prediction for the maximum of a hemisphere and the corresponding north-south asymmetry of a solar cycle may help to understand the mechanism of the solar cycle, the solar-terrestrial relationship, and solar-activity influences on space weather. Here we analysed the sunspot-group data from the Greenwich Photoheliographic Results (GPR) during 1874-1976 and Debrecen Photoheliographic Data (DPD) during 1977-2017 and studied the cycle-to-cycle variations in the values of 13-month smoothed monthly mean sunspot-group area in the whole sphere (WSGA), northern hemisphere (NSGA), and southern hemisphere (SSGA) at the epochs of maxima of Sunspot Cycles $12-24$ and at the epochs of maxima of WSGA, NSGA, and SSGA Cycles 1224 (note that solar-cycle variation of a parameter is expressed as a cycle of that parameter). The cosine fits to the values of WSGA, NSGA, and SSGA at the maxima of sunspot, WSGA, NSGA, and SSGA Cycles 12-24, and to the values of the corresponding north-south asymmetry, suggest the existence of $\mathrm{a} \approx 132$-year periodicity in the activity of the northern hemisphere, a 54-66year periodicity in the activity of the southern hemisphere, and a 50-66 year periodicity in the north-south asymmetry in activity at all the aforementioned epochs. By extrapolating the best-fit cosine curves we predicted the amplitudes and the corresponding north-south asymmetry of the 25th WSGA, NSGA, and SSGA cycles. We find that on average Solar Cycle 25 in sunspot-group area would be to some extent smaller than Solar Cycle 24 in sunspot-group area. However, by inputting the predicted amplitudes of the 25th WSGA, NSGA, and SSGA cycles in the linear relationship between sunspot-group area and sunspot number we find that the amplitude $(130 \pm 12)$ of Sunspot Cycle 25 would be
\end{abstract}

Bikasipura, Bengaluru-560 111, India

Formerly worked at Indian Institute of Astrophysics,

Bengaluru-560 034, India.

corref, email: jajj55@yahoo.co.in; email:jdotjavaraiah@gmail.

com; email: jj@iiap.res.in 
slightly larger than that of reasonably small Sunspot Cycle 24. Still it confirms that the beginning of the upcoming Gleissberg cycle would take place around Solar Cycle 25. We also find that except at the maximum of NSGA Cycle 25 where the strength of activity in the northern hemisphere would be dominant, the strength of activity in the southern hemisphere would be dominant at the maximum epochs of the 25th sunspot, WSGA, and SSGA cycles.

Keywords: Sun: Dynamo - Sun: Solar activity - Sun: Sunspots - (Sun:) Space Weather - (Sun:) Solar-Terrestrial relations

\section{Introduction}

The existence of differences between the strengths of solar activity in the Sun's northern and southern hemispheres is well known as the north-south asymmetry in solar-activity. It exists in most solar activity phenomena (Hathaway, 2015). Besides this, it is also known that the epochs of maxima of solar cycles are not the same in the northern and southern hemispheres, that is in some solar cycles the maximum in one of the hemispheres does not coincide with the maximum of the total (whole sphere/visible whole disk) activity (e.g. see Figure 1 in Javaraiah, 2020 and also see McIntosh et al., 2013). In some solar cycles the activity peak occurs first in the northern hemisphere and in some other cycles, it first occurs in the southern hemisphere. The existence of some systematic phase difference between the cycles of activity in the northern and southern hemispheres is also known (e.g. Zolotova et al., 2010; Norton and Gallagher, 2010; Muraközy and Ludmány, 2012; McIntosh et al., 2013; Ravindra, Chowdhury, and Javaraiah, $2021)$. There exist $\approx 12$-year, $\approx 55$-year, and $\approx 100$-year periodicities in northsouth asymmetry (Carbonell, Oliver, and Ballester, 1993; Verma, 1993; Javaraiah and Gokhale, 1997; Li et al., 2002; Deng et al., 2016; Javaraiah, 2020; Nagovitsyn, Osipova, and Nagovitsyna, 2021). However, the origin of these as well as the known short-term periodicities in the north-south asymmetry (e.g. Knaack, Stenflo, and Berdyugina, 2004; Ravindra and Javaraiah, 2015; Chowdhury et al., 2016; Mandal and Banerjee, 2016; Ravindra, Chowdhury, and Javaraiah, 2021) is not yet clear (Norton, Charbonneau, and Passos, 2014; Schüssler and Cameron, 2018 ; Nepomnyashchikh et al., 2019). Our recent analysis indicates that the $\approx 12$ year and $\approx 51$-year periodicities of north-south asymmetry are manifestations of the differences in strengths of the corresponding periodicities in northern and southern hemispheres and their origin might be connected to the configurations of giant planets (Javaraiah, 2020). It has been observed that in several solar cycles there exist some differences in the maximum epochs of sunspot number and sunspots area cycles (Hathaway, 2015). Recently, we have analysed the combined sunspot-group data from Greenwich Photoheliographic Results (GPR) during 1874-1976 and Debrecen Photoheliographic Data (DPD) during 1977-2017 and have predicted the north-south asymmetry in the average sunspot-group area at the maximum epoch of Sunspot Cycle 25 (Javaraiah, 2021). Here we analyse the aforementioned sunspot-group data and predict the north-south asymmetry at the maximum epochs of solar cycles in the northern and southern hemispheres. 
This may help in better understanding the solar dynamo and the solar-terrestrial relationship. Here our approach is different from that of Javaraiah (2021), where we have used the same method that was used in our earlier articles (Javaraiah, $2007 ; 2008 ; 2015)$. In those earlier articles the prediction was made based on the existence of a good correlation between the amplitude of a solar cycle and the sum of the areas of sunspot groups in the $0^{\circ}-10^{\circ}$ latitude interval of the southern hemisphere just after around one year from the maximum of the preceding cycle (hereafter area-sum). Here we study the cycle-to-cycle variations in the maxima of Solar Cycles 12-24 in the average areas of sunspot groups in northern and southern hemispheres, as well as in the whole sphere, by determining the best-fit cosine functions to these data. In principle, from this method it is possible to make predictions for several upcoming solar cycles. However, due to the considerable uncertainties in the obtained best-fit cosine functions, here we make cautious predictions for the amplitude and north-south asymmetry of Solar Cycle 25 only.

In the next section we describe the data analysis. In Section 3 we present the results, and in Section 4 we present the conclusions and briefly discuss them.

\section{Data Analysis}

Here the data and the analysis are the same as in Javaraiah (2019). In Javaraiah (2021) and here we have used the values of the amplitudes $\left(\left[R_{\mathrm{M}}\right]\right)$, i.e. the highest value of 13-month smoothed monthly mean sunspot number, and the maximum epochs of sunspot cycles given by Pesnell (2018). These were determined by Pesnell (2018) from the time series of 13-month smoothed monthly mean values of Version 2 international sunspot number (SN) available at www.sidc.be/silso/datafiles. The updated GPR (1874-1976) and DPD (1977-2017) daily sunspot-group data were downloaded from the website fenyi.solarobs.unideb.hu/pub/DPD/ (for details see Győri, Baranyi, and Ludmány, 2011; Baranyi, Győri, and Ludmány, 2016; Győri, Ludmány, and Baranyi, 2017). These data contain, besides the heliographic positions and other parameters, the corrected whole-spot area [ msh: millionth of solar hemisphere] of each sunspot group for its each day observation. First we determined the mean area of sunspot groups in the Sun's whole sphere (WSGA), northern hemisphere (NSGA), and southern hemisphere (SSGA) during each calender month of the years 1874-2017, and then we determined the 13 -month smoothed monthly mean values and the corresponding standard errors $(s=\sigma / \sqrt{13}$, where $\sigma$ is the standard deviation). From the time series of the 13-month smoothed monthly mean values we obtained the values and the corresponding epochs of the following parameters during SN, WSGA, NSGA, and SSGA Cycles 12-24 (note that for brevity and convenience solar cycle variation of a parameter is expressed as a cycle of that parameter).

$T_{\mathrm{M}}$ : epoch of SN cycle maximum,

$T_{\mathrm{W}}$ : epoch of WSGA cycle maximum,

$T_{\mathrm{N}}$ : epoch of NSGA cycle maximum,

$T_{\mathrm{S}}$ : epoch of SSGA cycle maximum,

It should be noted that usually in a solar cycle these epochs are not in an increasing or decreasing order. However rarely there could be an order, and even 
all these four epochs of a solar-cycle could be the same.

RWA: value of WSGA at $T_{\mathrm{M}}$,

RNA: value of NSGA at $T_{\mathrm{M}}$,

RSA: value of SSGA at $T_{\mathrm{M}}$,

WAM: value of maximum of a WSGA Cycle,

WNA: value of NSGA at $T_{\mathrm{W}}$,

WSA: value of SSGA at $T_{\mathrm{W}}$,

NAM: value of maximum of an NSGA Cycle,

NSA: value of SSGA at $T_{\mathrm{N}}$,

NWA: value of WSGA at $T_{\mathrm{N}}$,

SAM: value of maximum of an SSGA Cycle,

SNA: value of NSGA at $T_{\mathrm{S}}$, and

SWA: value of WSGA at $T_{\mathrm{S}}$.

We calculated a best-fit cosine function to the cycle-to-cycle modulation in each aforementioned parameter and by extrapolating the best-fit cosine curves we obtained the values of the above parameters for the 25th WSGA, NSGA, and SSGA cycles. We also calculated the best-fit cosine function to the cycle-tocycle modulation of relative north-south asymmetry $(N-S) /(N+S)$, where $N$ and $S$ represent the values of the parameters that correspond to the northern and southern hemispheres, respectively, at the epochs of maxima of sunspot, WSGA, NSGA, and SSGA cycle. We predict north-south asymmetry at the corresponding epochs of the 25 th cycles. In the calculations of the best-fit cosine functions, weights equal to the corresponding standard error of the parameters are used. All the linear least-squares fits were calculated by using the Interactive Digital Library (IDL) software FITEXY.PRO, which is downloaded from the website idlastro.gsfcnasa.gov/ftp/pro/math/. An advantage of using this software is that the errors in both abscissa and ordinate values will be taken into account in the calculation of linear fits by the least-squares method.

\section{Results and Predictions}

In Table 1, we have given the 13-month smoothed monthly mean values of WSGA, NSGA, and SSGA at the epochs of maxima of SN, WSGA, NSGA, and SSGA of Cycles 12-14. Figure 1 shows the values of the parameters NAM, SAM, WAM, and RWA given in this table versus time (respective epochs). The large (small) error bars in the values of these parameters of most of the large (small) solar cycles are due to the strong (weak) fluctuations in the monthly mean sunspot-group area around the maximum epochs of the large (small) solar cycles. In Table 2, we give the information obtained from this figure and Table 1 about dominant hemisphere, phase-leading hemisphere, and the hemisphere coinciding with the whole sphere. In the same table, the difference between the epochs of $R_{\mathrm{M}}$ and WAM is also given. As we can see in Figure 1 and Table 2 in some solar cycles North is dominant and in some other cycles South is dominant. In many solar cycles the maximum in one of the hemispheres does not coincide with the maximum of the total (whole sphere) activity. In some solar cycles the activity 
Table 1 . The values $[\mathrm{msh}]$ of WSGA, NSGA, and SSGA at the epochs $T_{\mathrm{M}}, T_{\mathrm{W}}, T_{\mathrm{N}}$, and $T_{\mathrm{S}}$ of the maxima of SN, WSGA, NSGA, and SSGA cycles $(n=12,13, \ldots, 24)$, respectively, determined from the corresponding time series of 13-month smoothed monthly mean areas of the sunspot groups in whole sphere, northern hemisphere, and southern hemisphere. The maximum $\left[R_{\mathrm{M}}\right]$ values of $\mathrm{SN}$ are also given (all the maximum values are indicated with bold).

\begin{tabular}{|c|c|c|c|c|c|c|c|c|c|}
\hline \multirow[b]{2}{*}{$n$} & \multirow[b]{2}{*}{$T_{\mathrm{M}}$} & \multicolumn{4}{|c|}{ At maximum $R_{\mathrm{M}}$ of $\mathrm{SN}$} & \multirow[b]{2}{*}{$T_{\mathrm{W}}$} & \multicolumn{3}{|c|}{ At maximum WAM of WSGA } \\
\hline & & $R_{\mathrm{M}}$ & RWA & RNA & RSA & & WAM & WNA & WSA \\
\hline 12 & 1883.96 & $124.4 \pm 12.5$ & $1371 \pm 122$ & $414 \pm 75$ & $957 \pm 105$ & 1883.96 & $1371 \pm 122$ & $414 \pm 75$ & $957 \pm 105$ \\
\hline 13 & 1894.04 & $146.5 \pm 10.8$ & $1616 \pm 110$ & $621 \pm 57$ & $995 \pm 100$ & 1894.04 & $1616 \pm 110$ & $621 \pm 57$ & $995 \pm 100$ \\
\hline 14 & 1906.12 & $107.1 \pm 9.2$ & $1044 \pm 140$ & $761 \pm 139$ & $283 \pm 43$ & 1905.45 & $1161 \pm 158$ & $745 \pm 137$ & $416 \pm 90$ \\
\hline 15 & 1917.62 & $175.7 \pm 11.8$ & $1535 \pm 171$ & $829 \pm 124$ & $707 \pm 89$ & 1917.54 & $1554 \pm 165$ & $853 \pm 123$ & $701 \pm 92$ \\
\hline 16 & 1928.29 & $130.2 \pm 10.2$ & $1324 \pm 123$ & $631 \pm 96$ & $693 \pm 65$ & 1926.29 & $1467 \pm 211$ & $809 \pm 181$ & $658 \pm 98$ \\
\hline 17 & 1937.29 & $198.6 \pm 12.6$ & $2120 \pm 176$ & $1309 \pm 140$ & $811 \pm 108$ & 1937.29 & $2120 \pm 176$ & $1309 \pm 140$ & $811 \pm 108$ \\
\hline 18 & 1947.37 & $218.7 \pm 10.3$ & $2641 \pm 210$ & $1051 \pm 121$ & $1590 \pm 237$ & 1947.37 & $2641 \pm 210$ & $1051 \pm 121$ & $1590 \pm 237$ \\
\hline 19 & 1958.20 & $285.0 \pm 11.3$ & $3441 \pm 208$ & $1749 \pm 164$ & $1693 \pm 144$ & 1957.96 & $3480 \pm 239$ & $1801 \pm 153$ & $1679 \pm 166$ \\
\hline 20 & 1968.87 & $156.6 \pm 8.4$ & $1556 \pm 82$ & $951 \pm 83$ & $605 \pm 46$ & 1970.54 & $1628 \pm 93$ & $926 \pm 70$ & $701 \pm 121$ \\
\hline 21 & 1979.96 & $232.9 \pm 10.2$ & $2121 \pm 162$ & $1064 \pm 139$ & $1057 \pm 142$ & 1981.71 & $2338 \pm 177$ & $1065 \pm 134$ & $1274 \pm 193$ \\
\hline 22 & 1989.87 & $212.5 \pm 12.7$ & $2269 \pm 193$ & $1121 \pm 124$ & $1148 \pm 118$ & 1989.45 & $2591 \pm 179$ & $1401 \pm 141$ & $1190 \pm 126$ \\
\hline 23 & 2001.87 & $180.3 \pm 10.8$ & $2157 \pm 206$ & $1073 \pm 111$ & $1084 \pm 152$ & 2002.20 & $2334 \pm 179$ & $951 \pm 114$ & $1383 \pm 141$ \\
\hline \multirow[t]{3}{*}{24} & 2014.29 & $116.4 \pm 8.2$ & $1600 \pm 116$ & $420 \pm 40$ & $1180 \pm 144$ & 2014.45 & $1629 \pm 114$ & $418 \pm 40$ & $1211 \pm 143$ \\
\hline & & \multicolumn{3}{|c|}{ At maximum NAM of NSGA } & & \multicolumn{3}{|c|}{ At maximum SAM of SSGA } & \\
\hline & $T_{\mathrm{N}}$ & NWA & NAM & NSA & $T_{\mathrm{S}}$ & SWA & SNA & SAM & \\
\hline 12 & 1882.37 & $978 \pm 145$ & $476 \pm 72$ & $501 \pm 116$ & 1883.96 & $1371 \pm 122$ & $414 \pm 75$ & $957 \pm 105$ & \\
\hline 13 & 1894.12 & $1585 \pm 115$ & $649 \pm 61$ & $935 \pm 115$ & 1893.96 & $1585 \pm 111$ & $577 \pm 55$ & $1008 \pm 99$ & \\
\hline 14 & 1906.04 & $1109 \pm 143$ & $822 \pm 135$ & $287 \pm 45$ & 1907.45 & $1098 \pm 129$ & $512 \pm 81$ & $585 \pm 103$ & \\
\hline 15 & 1917.71 & $1492 \pm 178$ & $855 \pm 116$ & $638 \pm 84$ & 1917.62 & $1535 \pm 171$ & $829 \pm 124$ & $707 \pm 89$ & \\
\hline 16 & 1926.29 & $1467 \pm 211$ & $809 \pm 181$ & $658 \pm 98$ & 1928.21 & $1338 \pm 121$ & $584 \pm 102$ & $754 \pm 57$ & \\
\hline 17 & 1937.54 & $2088 \pm 195$ & $1374 \pm 158$ & $714 \pm 109$ & 1938.62 & $1855 \pm 164$ & $715 \pm 91$ & $1141 \pm 151$ & \\
\hline 18 & 1949.62 & $2060 \pm 196$ & $1200 \pm 114$ & $860 \pm 153$ & 1947.45 & $2625 \pm 215$ & $1011 \pm 129$ & $1614 \pm 232$ & \\
\hline 19 & 1959.54 & $2847 \pm 233$ & $2220 \pm 227$ & $627 \pm 77$ & 1957.79 & $3428 \pm 257$ & $1673 \pm 183$ & $1755 \pm 142$ & \\
\hline 20 & 1967.62 & $1596 \pm 142$ & $1108 \pm 100$ & $488 \pm 73$ & 1969.87 & $1549 \pm 113$ & $767 \pm 103$ & $783 \pm 105$ & \\
\hline 21 & 1979.37 & $2067 \pm 164$ & $1211 \pm 111$ & $856 \pm 117$ & 1981.96 & $2293 \pm 199$ & $976 \pm 123$ & $1317 \pm 179$ & \\
\hline 22 & 1989.45 & $2591 \pm 179$ & $1401 \pm 141$ & $1190 \pm 126$ & 1991.54 & $2405 \pm 167$ & $894 \pm 129$ & $1511 \pm 189$ & \\
\hline 23 & 2000.96 & $1831 \pm 167$ & $1122 \pm 119$ & $709 \pm 65$ & 2002.20 & $2334 \pm 179$ & $951 \pm 114$ & $1383 \pm 141$ & \\
\hline 24 & 2011.71 & $905 \pm 116$ & $687 \pm 100$ & $218 \pm 40$ & 2014.45 & $1629 \pm 114$ & $418 \pm 40$ & $1211 \pm 143$ & \\
\hline
\end{tabular}

peak occurs first in the northern hemisphere and in some other cycles, it occurs first in the southern hemisphere.

In the case of sunspot number, the amplitude of Solar Cycle 21 is larger than that of Solar Cycle 22, whereas, as can be seen in Figure 1, in the case of sunspot area the behavior is opposite to that of sunspot number, i.e. Solar Cycle 22 is larger than Solar Cycle 21 (Hathaway, 2015). This difference could be because the ratio of small to large numbers of sunspots/sunspot groups may be larger in Solar Cycle 21 than in Solar Cycle 22. The aforementioned opposite behavior is also found in the cases of whole sphere, northern, and southern hemispheres' mean areas of sunspot groups (i.e. RWA, RNA, and RSA here) at the epochs of maxima of Solar Cycles 21 and 22 (see also Javaraiah, 2019). As we can see in Table 1, except in the case of SNA, this property exists in the case of all remaining parameters. The value of SNA of SSGA Cycle 21 is larger than that of SSGA Cycle 22.

According to the Gnevyshev-Ohl rule (G-O: Gnevyshev and Ohl, 1948), an odd-numbered sunspot cycle is larger than its preceding even-numbered sunspot cycle. However, sunspot-cycle pair $(22,23)$ violated this rule, i.e. $R_{\mathrm{M}}$ of Sunspot 


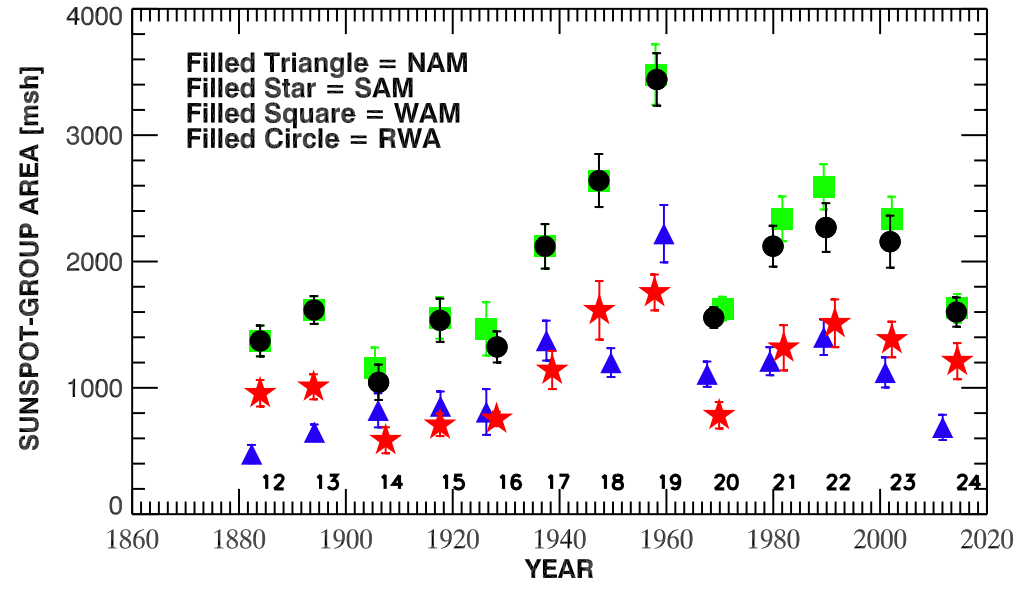

Figure 1. Values given in Table 1 versus time (corresponding epochs) of the parameters: NAM, i.e. the values of maxima of NSGA Cycles 12-24; SAM, i.e. the values of maxima of SSGA Cycles $12-24$; WAM, i.e. the values of maxima of WSGA Cycles $12-24$; and RWA, i.e. the values of the 13 -month smoothed monthly mean sunspot-group areas at the maxima of Sunspot Cycles $12-24$.

Table 2. The information obtained by using Table 1 and Figure 1 about dominant hemisphere $(\mathrm{DH})$, phase-leading hemisphere (LH), and whether the hemisphere coincides/is close to the whole sphere $(\mathrm{CH})$ in a solar cycle $(n)$. The corresponding differences between the values of parameters and between the epochs are given within parentheses. In the last column the differences between $T_{\mathrm{M}}$ and $T_{\mathrm{W}}$ are also given. In the $2 \mathrm{nd}$ column the "same" implies that there is no phase difference. In the 4th and 5th columns a zero/small absolute value indicates that the corresponding peaks are coincident/very close.

\begin{tabular}{ccccc}
\hline$n$ & DH (NAM-SAM) & LH $\left(T_{\mathrm{N}}-T_{\mathrm{S}}\right)$ & CH $\left(T_{\mathrm{N}}-T_{\mathrm{W}}\right) /\left(T_{\mathrm{S}}-T_{\mathrm{W}}\right)$ & $T_{\mathrm{M}}-T_{\mathrm{W}}$ \\
\hline 12 & south $(-481)$ & north $(-1.59)$ & south $(-1.59 / 0.0)$ & 0.0 \\
13 & south (-359) & same (0.16) & both $(0.08 /-0.08)$ & 0.0 \\
14 & north (237) & north (-1.4) & north $(0.59 / 2.0)$ & -0.67 \\
15 & north (148) & same (0.09) & both $(0.17 / 0.08)$ & -0.08 \\
16 & north (55) & north (-1.92) & north $(0.0 / 1.92)$ & -2.0 \\
17 & north (233) & north (-1.08) & north $(0.25 / 1.33)$ & 0.0 \\
18 & south (-414) & south (2.17) & south $(2.25 / 0.08)$ & 0.0 \\
19 & north (465) & south (1.75) & south $(1.58 /-0.17)$ & -0.24 \\
20 & north (325) & north (-2.25) & south $(-2.92 /-0.67)$ & 1.67 \\
21 & south (-106) & north (-2.59) & south $(-2.34 / 0.25)$ & 1.75 \\
22 & south (-110) & north (-2.09) & north $(0.0 / 2.09)$ & -0.42 \\
23 & south (-261) & north (-1.24) & south $(-1.24 / 0.0)$ & 0.33 \\
24 & south (-524) & north $(-2.74)$ & south $(-2.74 / 0.0)$ & 0.16 \\
\hline
\end{tabular}



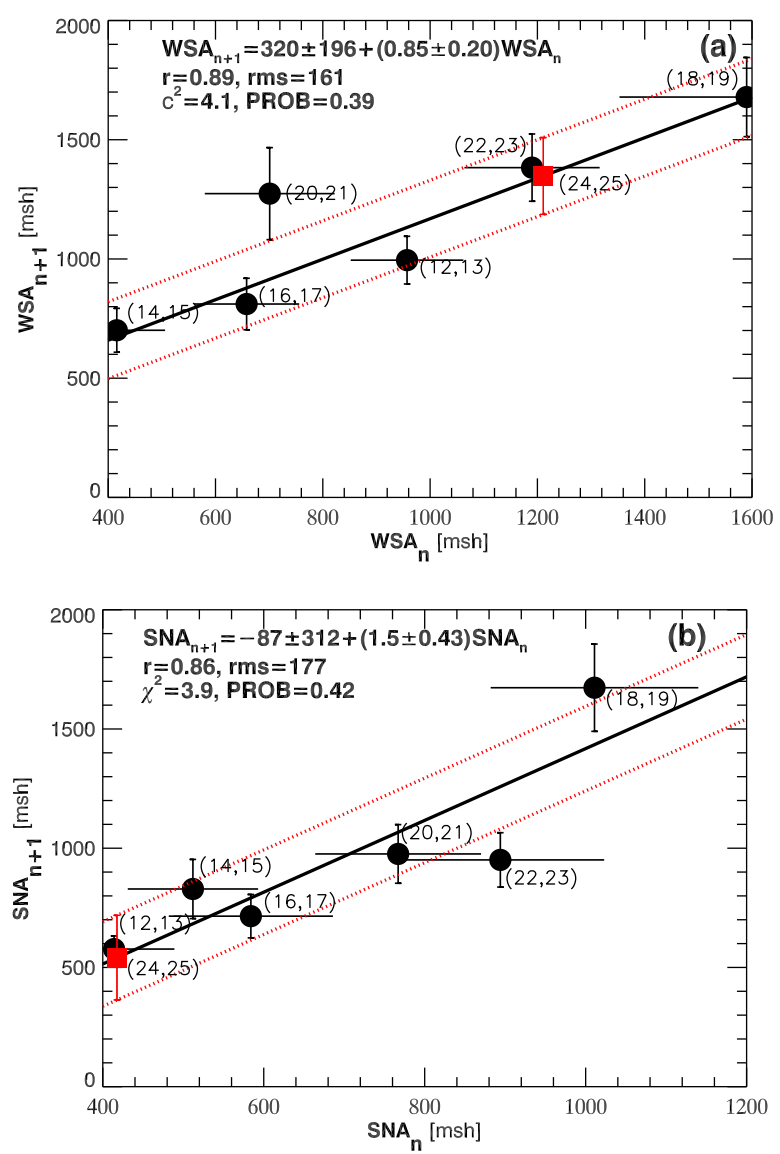

Figure 2. (a) $\mathrm{WSA}_{n}$ of an even-numbered WSGA cycle versus $\mathrm{WSA}_{n+1}$ of following odd-numbered WSGA cycle and (b) $\mathrm{SNA}_{n}$ of an even-numbered SSGA cycle versus $\mathrm{SNA}_{n+1}$ of following odd-numbered SSGA cycle. The horizontal and vertical error bars represent the standard errors of the abscissa and the ordinate, respectively; both are taken into account in the calculations of linear least-square fits. The continuous line represents the best-fit linear relationship. The dotted lines (red) are drawn at one-rms (root-mean-square deviation) levels. The best-fit linear equation and the values of correlation coefficient $[r], \chi^{2}$ and the corresponding probability (PROB), and rms are also shown. The filled squares (red) represent the predicted values of WSA and SNA at the epochs of maxima of WSGA Cycle 25 and SSGA Cycle 25 .

Cycle 22 is larger than that of Sunspot Cycle 23. As we can see in Table 1, WSA values of WSGA cycle pair $(22,23)$ and SNA values of SSGA cycle pair $(22$, 23) satisfied the $\mathrm{G}-\mathrm{O}$ rule and the corresponding pairs of each of the remaining all parameters violated the $\mathrm{G}-\mathrm{O}$ rule (here the $\mathrm{G}-\mathrm{O}$ rule in WSA and SNA tentatively means that the cycle-to-cycle modulations in WSA and SNA imitate the $\mathrm{G}-\mathrm{O}$ rule of solar cycles).

Figure 2 shows the linear relationships between WSA pairs of even- and oddnumbered WSGA cycles and between SNA pairs of even- and odd-numbered SSGA cycles. Besides the obtained linear equation, the values of $\chi^{2}$ and the 
corresponding probabilities (PROB), and rms (root-mean-square deviation) are also shown in Figure 2. The probabilities of the corresponding values of $\chi^{2}$ of the best-fit linear relationships are somewhat small. Note that the $\chi^{2}$-probability is a scalar (between zero and unity) giving the probability that a correct model would give a value equal or larger than the observed $\chi^{2}$. A small value of PROB indicates a poor fit, perhaps because the errors are underestimated.

By using the linear relationships shown in Figure 2 we get the values $1348 \pm 161$ msh and $542 \pm 177$ msh for WSA of WSGA Cycle-25 and SNA of SSGA Cycle 25 , respectively. These values are larger than the corresponding observed values of WSGA Cycle 24 and SSGA Cycle 24 implying satisfaction of the G-O rule. By using the predicted value of WSA and the existence of reasonably good correlations (good linear relationships) between WSA and SAM, SAM and SWA, SWA and WAM, WAM and RWA, and RWA and $R_{\mathrm{M}}$ we obtained the values for WAM of WSGA Cycle 25 and $R_{\mathrm{M}}$ of Sunspot Cycle 25, which were found to be much larger than the corresponding values of the respective 24 th cycles. We have not given them here because the aforementioned predicted values of WSA of WSGA Cycle 25 and SNA of SSGA Cycle 25 are to some extent unreliable. It should be noted that without prior knowledge about non-violation of the $\mathrm{G}-\mathrm{O}$ rule by an upcoming even- and odd-numbered solar cycle pair, by using the $\mathrm{G}-\mathrm{O}$ rule it is not possible to predict the amplitude of the odd-numbered solar cycle.

We calculated the cosine fits to the values of the parameters of WSGA, NSGA, and SSGA Cycles $12-24$ given in Table 1. In Figures 3-6, we show the best-fit cosine curves. In Table 3, we give the results obtained by extrapolating the bestfit cosine curves of different parameters, i.e. the value of period, the predicted corresponding 25th cycle value of a parameter, and the values of rms and $\chi^{2}$. The cosine fit of each parameter seems to be reasonably good because only one (mostly the data point of Cycle 19) or at most two data points are outliers (away from the one-rms level). In principle, we can extrapolate the best-fit cosine curves for several cycles. However, the $\chi^{2}$ values are large, i.e. the cosine best fits are not very accurate. Therefore the corresponding results are only suggestive rather than compelling. Hence we restricted our conclusions to only the predictions for the corresponding 25th cycle (although the values obtained for the corresponding 26th cycle are also shown in Figures 3-6).

We can see in Table 3 that the periodicity in a parameter of the northern hemisphere is $\approx 132$ years, whereas it is $54-66$ years in a parameter of the southern hemisphere, i.e. approximately half of that of the northern hemisphere. This is consistent with the similar results noticed in the earlier analysis (Javaraiah, 2019 ). Morlet-wavelet analysis were also suggested that a $\approx 51$-year periodicity in the 13-month smoothed area of sunspot groups of the northern hemisphere is much weaker than that of the southern hemisphere (Javaraiah, 2020).

The cosine fits to the data of RWA, WAM, NWA, and SWA are found to be much more uncertain, and therefore we do not show them. We get the corresponding 25 th cycle values of these parameters from the predicted values of the parameters given in Table 3 .

$\mathrm{RWA}=\mathrm{RNA}+\mathrm{RSA}=1317 \pm 123 \mathrm{msh}$,

$\mathrm{WAM}=\mathrm{WNA}+\mathrm{WSA}=1402 \pm 124 \mathrm{msh}$,

$\mathrm{NWA}=\mathrm{NAM}+\mathrm{NSA}=1082 \pm 100 \mathrm{msh}$,

$\mathrm{SWA}=\mathrm{SNA}+\mathrm{SAM}=1354 \pm 116 \mathrm{msh}$. 



Figure 3. Continuous curve represents the best-fit cosine function to the values (filled circles) (a) of RNA and (b) of RSA at the epochs of maxima of Sunspot Cycles 12 - 24, i.e the values of NSGA and SSGA at $T_{\mathrm{M}}$. The dotted curve (red) represents the one-rms level. The extrapolated portion is shown as a dashed curve and the filled squares (red) represent the predicted values of RNA and RSA at the maximum epochs of Sunspot Cycles 25 and 26. The period (in number of solar cycles) of the cosine function is also shown. See also the details given in Table 3 .
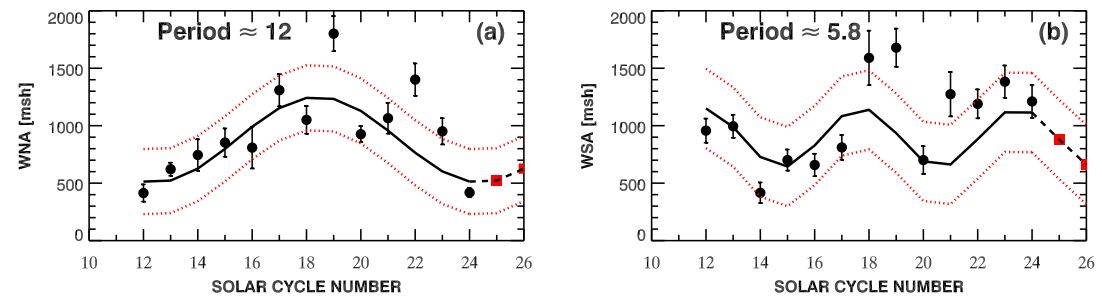

Figure 4. Continuous curve represents the best-fit cosine function to the values (filled circles) (a) of WNA and (b) of WSA of WSGA Cycles 12-24, i.e. the values of NSGA and SSGA at $T_{\mathrm{W}}$. The dotted curve (red) represents the one-rms level. The extrapolated portion is shown as a dashed curve and the filled squares (red) represent the predicted values of WNA and WSA at the maximum epochs of WSGA Cycles 25 and 26. The period (in number of solar cycles) of the cosine function is also shown. See also the details given in Table 3.

The rms appears large for the corresponding predicted value of a parameter of Solar Cycle 25 given Table 3. However, the range of values of the parameter is large and the predicted value is at the minimum level of the corresponding longterm cycle (Gleissberg cycle). The uncertainties in the values of RWA, WAM, NWA, and SWA obtained above are reasonably small. They are determined as $\sqrt{\left(\mathrm{rms}_{\mathrm{N}}^{2}+\mathrm{rms}_{\mathrm{S}}^{2}\right) / 13}$, where $\mathrm{rms}_{\mathrm{N}}$ and $\mathrm{rms}_{\mathrm{S}}$ are the rms values of northern and southern hemispheres' parameters, respectively.

We also determined the best-fit cosine function to the values of $R_{\mathrm{M}}$ of SN Cycles 12-24. It is shown in Figure 7, and the details are also given in the last row of Table 3 . The obtained value for $R_{\mathrm{M}}$ of SN Cycle 25 is slightly larger than that of SN Cycle 24, but the former has a large uncertainty (rms value). Moreover, the cosine fit of $R_{\mathrm{M}}$ is not good, since the corresponding $\chi^{2}$ is very large.

Figure 8 shows the correlation between WAM and RWA. Obviously, there exists a high correlation between WAM and RWA. The corresponding linear least-squares best-fit is highly statistically significant $\left(\chi^{2}=2, \mathrm{PROB}=0.998\right)$. Using the above predicted value of WAM in WAM-RWA relationship shown in Figure 8, we get the value $1348 \pm 96$ msh for RWA of SN Cycle 25, which seems to be more reliable than the value of RWA obtained above by summing 

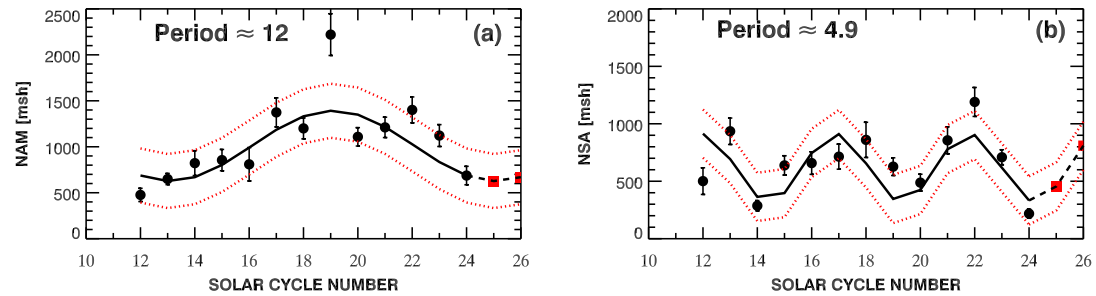

Figure 5. Continuous curve represents the best-fit cosine function to the values (filled circles) (a) NAM, i.e. the maximum values of NSGA Cycles 12-24 and (b) NSA, i.e. the values of SSGA at $T_{\mathrm{N}}$. The dotted curve (red) represents the one-rms level. The extrapolated portion is shown as a dashed curve and the filled squares (red) represent the predicted values of NAM and NSA at the maximum epochs of NSGA Cycles 25 and 26. The period (in number of solar cycles) of the cosine function is also shown. See also the details given in Table 3.


Figure 6. Continuous curve represents the best-fit cosine function to the values (filled circles) (a) SNA, i.e. the values of NSGA at $T_{\mathrm{S}}$, and (b) SAM, i.e. the maximum values of SSGA Cycles $12-24$. The dotted curve (red) represents the one-rms level. The extrapolated portion is shown as a dashed curve and the filled squares (red) represent the predicted values of SNA and SAM at the maximum epochs of SSGA Cycles 25 and 26. The period (in number of solar cycles) of the cosine function is also shown. See also the details given in Table 3.

the values predicted for RNA and RSA (note that the value of $\chi^{2}$ corresponding to the best-fit cosine function of RSA is very high). However, the two values are almost equal.

In Figure 9 we compare the predicted values of the parameters at the epochs of the maxima of the 25th SN, WSGA, NSGA, and SSGA cycles with the observed values of the corresponding 24th cycles. As we can see in this figure there is an

Figure 7: Continuous curve represents the best-fit cosine function to the values (filled circles) of $R_{\mathrm{M}}$, i.e. the values of the amplitudes of Sunspot Cycles 12-24. The dotted curve (red) represents the one-rms level. The extrapolated portion is shown as a dashed curve and the filled squares (red) represent the predicted values of $\mathrm{SN}$ at the epochs of maxima of Sunspot Cycles 25 and 26. The period (in number of solar cycles) of the cosine function is also shown. See also the details given in


Table 3. 


\begin{tabular}{lccccc}
\hline Parameter & $\begin{array}{c}\text { Period } \\
{[\text { cycles }]}\end{array}$ & $\begin{array}{c}\text { Period } \\
{[\text { years }]}\end{array}$ & Pred. Cycle 25 & rms & $\chi^{2}$ \\
\hline RNA & $\approx 12$ & $\approx 132$ & 491 & 255 & 66 \\
RSA & $\approx 6$ & $\approx 66$ & 826 & 358 & 118 \\
WNA & $\approx 12$ & $\approx 132$ & 522 & 283 & 69 \\
WSA & $\approx 5.8$ & $\approx 64$ & 880 & 345 & 69 \\
NAM & $\approx 12$ & $\approx 132$ & 627 & 294 & 46 \\
NSA & $\approx 4.9$ & $\approx 54$ & 455 & 210 & 64 \\
SNA & $\approx 12$ & $\approx 132$ & 461 & 242 & 47 \\
SAM & $\approx 5.5$ & $\approx 61$ & 893 & 342 & 67 \\
$R_{\mathrm{M}}$ & $\approx 12.3$ & $\approx 136$ & 123 & 33 & 141 \\
\hline
\end{tabular}

Table 3: The periods of bestfit cosine functions of the different parameters given in Table 1 and the parameter values ( $\mathrm{msh}$, in the case of a parameter of area) in Solar Cycle 25 predicted by extrapolating the best-fit cosine curves. The corresponding values of $\mathrm{rms}$ and $\chi^{2}$ are also given. Note that the average period of solar cycles is $11.03 \pm 1.18$-year (Pesnell, 2018.)

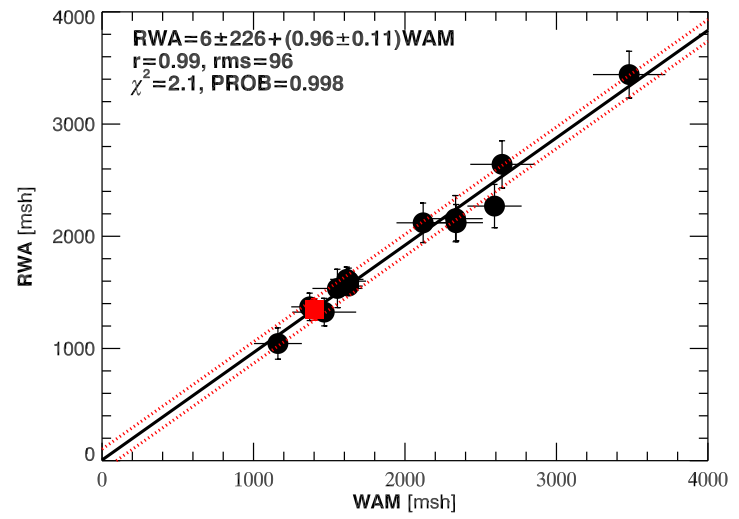

Figure 8: Scatter plot of RWA during Sunspot Cycles 12-24 versus WAM during WSGA Cycles $12-24$. The continuous line represents the linear least-square bestfit to the data. The dotted line (red) represents the one-rms level. The obtained linear equation and values of the corresponding $r$, rms, $\chi^{2}$, and PROB are given. The filled square (red) represents the predicted value of RWA, i.e. the value of WSGA at the epoch of $R_{\mathrm{M}}$ of Solar Cycle 25.

indication that except at the peaks of both the NSGA Cycles 24 and 25 where the activity in the northern hemisphere looks to be negligibly larger than that in southern hemisphere, in all the remaining other occasions, including at the epoch of $R_{\mathrm{M}}$, the southern hemisphere is dominant. The difference between the predicted values of all northern and southern hemispheres' parameters of the 25th cycles at a given epoch is not significant with respect to the large uncertainties in

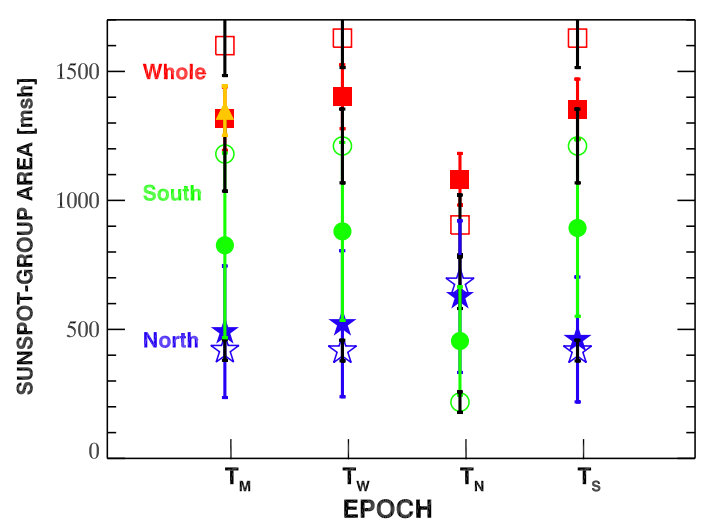

Figure 9: predicted values of the mean sunspot-group area at $T_{\mathrm{M}}, T_{\mathrm{W}}, T_{\mathrm{N}}$, and $T_{\mathrm{S}}$, i.e. at the epochs of maxima of the 25th SN, WSGA, NSGA, and SSGA cycles, respectively (filled symbol) and the corresponding observed values of the 24 th cycles (open symbol) versus the maximum epochs (the differences between the epochs of $X$-axis are not equal). The symbols square, star, and circle represent symbols square, star, and circle represent
the corresponding WSGA, NSGA and SSGA values, respectively. First column: RWA, RNA, and RSA at the epochs of maximum of SN Cycles 24 and 25, Second column: WAM, WNA, and WSA at the maximum of WSGA Cycles 24 and 25, Third column: NWA, NAM, and NSA at the maximum of NSGA Cycles 24 and 25, and Fourth column: SWA, SNA, and SAM at the maximum of SSGA Cycles 24 and 25 . The filled triangle represents the value of RWA obtained from the WAM-RWA relationship shown in Figure 8. 
Figure 10: Scatter plot of 13month smoothed monthly mean northern hemisphere's sunspot number $\left(\mathrm{SN}_{\mathrm{N}}\right)$ versus NSGA during the period 1992-2017 (300 data points). The continuous line (red) represents the linear least-squares best-fit to the data. The dotted line (red) represents the one-rms level. The obtained linear equation and the values of the corresponding $r, \mathrm{rms}, \chi^{2}$, and $\mathrm{PROB}$ are given. The predicted values of $\mathrm{SN}_{\mathrm{N}}$ at the epochs of $R_{\mathrm{M}}$, WAM, NAM, and NSA of the 25th SN, WSGA, NSGA, and SSGA cycles are also shown.

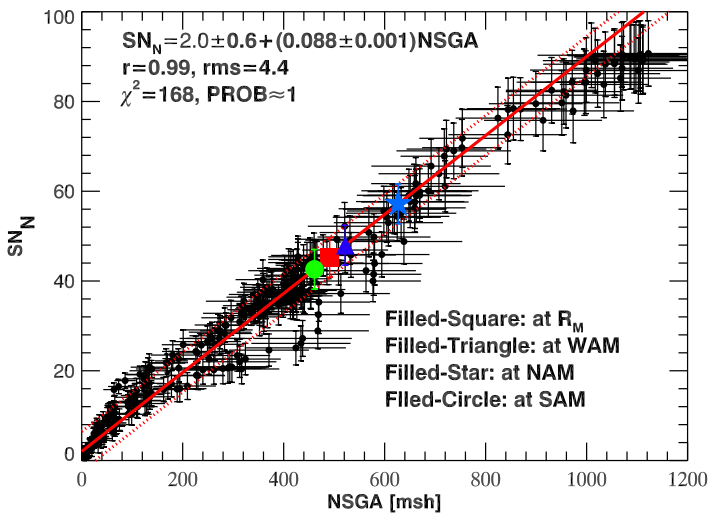

Figure 11: Scatter plot of 13month smoothed monthly mean southern hemisphere's sunspot number $\left(\mathrm{SN}_{\mathrm{S}}\right)$ versus SSGA during the period 1992-2017 (300 data points). The continuous line (red) represents the linear least-squares best fit to the data. The dotted line (red) represents the one-rms level. The obtained linear equation and the values of the corresponding $r$, rms, $\chi^{2}$, and PROB are given. The predicted values of $\mathrm{SN}_{\mathrm{S}}$ at the epochs of $R_{\mathrm{m}}$, WAM, SNA, and SAM of 25th SN, WSGA, NSGA, and SSGA

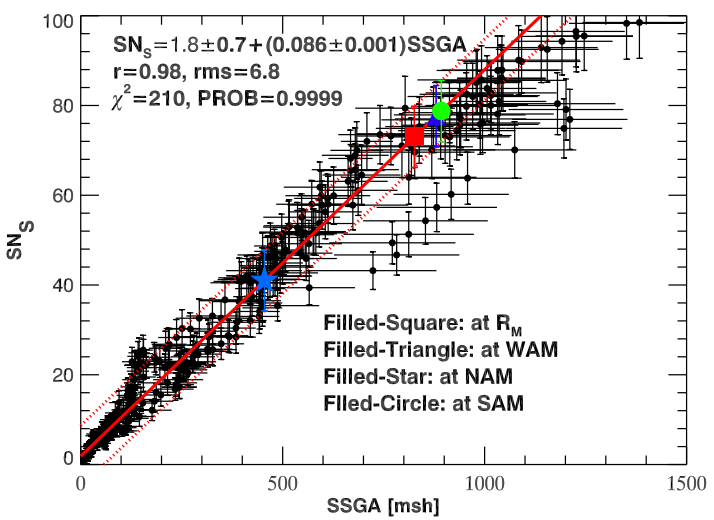
cycles are also shown.

these values, suggesting that there exists no significant north-south asymmetry in Solar Cycle 25, whereas most of the corresponding differences of the 24th cycle seem to be statistically significant. The overall pattern of Solar Cycle 25 would be closely similar to that of Solar Cycle 24 . The value predicted here for RWA is much larger than the value $\approx 701 \mathrm{msh}$ of RWA (i.e. $A_{\mathrm{W}}$ ) predicted by Javaraiah (2021) by a different method, which is much smaller than the RWA of Solar Cycle 24. The above predicted values of RWA and WSA are reasonably smaller than the corresponding values of Solar Cycle 24, and there are no significant differences in the corresponding values of WNA. The predicted value of WAM of Solar Cycle 25 is also slightly smaller than that of Solar Cycle 24, suggesting that on average Solar Cycle 25 in sunspot-group area would be smaller than the corresponding Solar Cycle 24. However, the uncertainties in the predicted values of Solar Cycle 25 are large. Therefore all the above results are only suggestive rather than compelling.

As shown in Figures 5-7 of Javaraiah (2021), here we also attempted to determine the linear relations between RWA and $R_{\mathrm{M}}$, RWA and RNA, and 

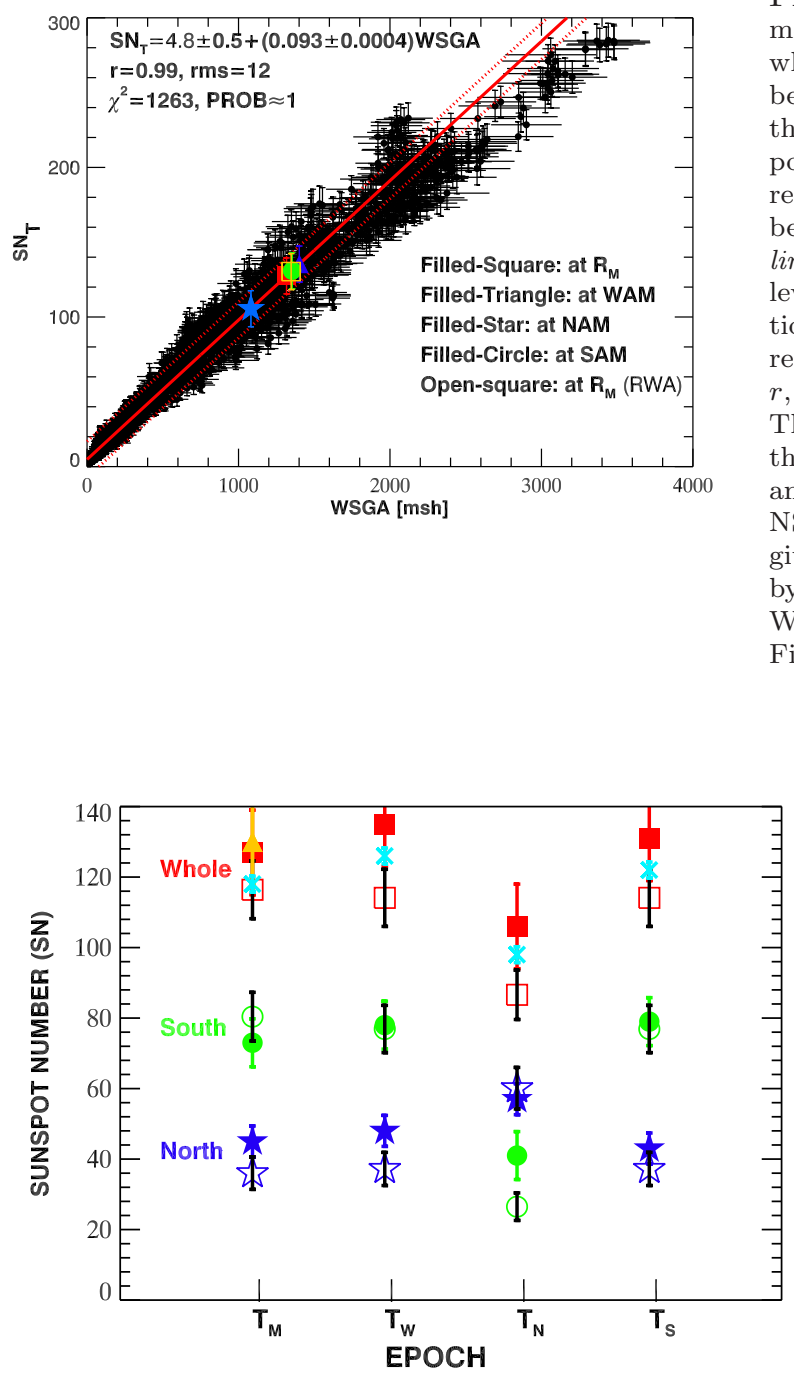

Figure 12: Scatter plot of 13month smoothed monthly mean whole-sphere (total) sunspot number $\left(\mathrm{SN}_{\mathrm{T}}\right)$ versus WSGA during the period 1874-2017 (1713 data points). The continuous line (red) represents the linear least-squares best-fit to the data. The dotted line (red) represents the one-rms level. The obtained linear equation and the values of the corresponding correlation coefficient $r, \mathrm{rms}, \chi^{2}$, and PROB are given. The predicted values of $\mathrm{SN}_{\mathrm{T}}$ at the epochs of $R_{\mathrm{M}}$, WAM, NAM, and SAM of the 25th SN, WSGA, NSGA, and SSGA cycles are also given. The value of $\mathrm{SN}_{\mathrm{T}}$ obtained by using RWA predicted from the WAM-RWA relationship shown in Figure 8 is also given.

Figure 13: Predicted values of SN given in Table 4 at $T_{\mathrm{M}}, T_{\mathrm{W}}, T_{\mathrm{N}}$, and $T_{\mathrm{S}}$, i.e. at the epochs of maxima of SSGA SSGA cycle, respectively (filled symbols) and the observed values of $\mathrm{SN}$ of the corresponding 24 th cycles (open symbols) versus the maximum epochs (the differences between the epochs of $X$-axis are not equal). The symbols square, star, and circle represent $\mathrm{SN}_{\mathrm{T}}, \mathrm{SN}_{\mathrm{N}}$, and $\mathrm{SN}_{\mathrm{S}}$ values, respectively (note that $\mathrm{SN}_{\mathrm{T}}$ at $T_{\mathrm{M}}$ is the same as $R_{\mathrm{M}}$ ). The first, second, third, and fourth columns contain the values of $\mathrm{SN}_{\mathrm{T}}, \mathrm{SN}_{\mathrm{N}}$, and $\mathrm{SN}_{\mathrm{S}}$ at the maximum epochs of $\mathrm{SN}_{\mathrm{T}}$ WSGA, NSGA, and SSGA Cycles 24 and 25 , SSctivelycles 24 and 25 , respectively. The $\mathrm{SN}_{\mathrm{T}}$ a represents the value of $\mathrm{SN}_{\mathrm{T}}$ obtained by summing the predicted values of $\mathrm{SN}_{\mathrm{N}}$ and $\mathrm{SN}_{S}$. The filled triangle represents the value of $R_{\mathrm{M}}$ obtained by using the value of RWA obtained from WAMRWA relationship shown in Figure 8

RWA and RSA. Here in the calculations of linear least-squares fit the errors in the values of both abscissa and ordinate are taken into account, whereas in that earlier paper, only errors in the ordinate values were considered. None of the corresponding linear least-squares best fits was found to be reasonably statistically significant, that is, the corresponding values of $\chi^{2}$ are found to be very large and, obviously the corresponding values of PROB are found to be very small $(\leq 0.07)$. If we use in Equation 5 of Javaraiah $(2021)$ the values $\approx 1317 \mathrm{msh}$ and $\approx 1348 \mathrm{msh}$ obtained/predicted above for RWA (i.e. $A_{\mathrm{W}}$ in that paper), then we get $129 \pm 19$ and $131 \pm 19$, respectively, for $R_{\mathrm{M}}$ of Sunspot Cycle 25. Each of 
Table 4. Predictions for the values of SN in northern hemisphere $\left(\mathrm{SN}_{\mathrm{N}}\right)$, southern hemisphere $\left(\mathrm{SN}_{\mathrm{S}}\right)$, and whole sphere $\left(\mathrm{SN}_{\mathrm{T}}\right)$ at the epochs $T_{\mathrm{M}}, T_{\mathrm{W}}, T_{\mathrm{N}}$, and $T_{\mathrm{S}}$ of the maxima of 25 th SN, WSGA, NSGA, and SSGA cycles, respectively, by using in the linear relationships shown in Figures 10-12 the values of the different parameters predicted from their respective best-fit cosine functions. The symbol a indicates that the value of RWA is obtained from WAM-RWA relationship shown in Figure 8 (note that $\mathrm{SN}_{\mathrm{T}}$ at $T_{\mathrm{M}}$ is the same as $R_{\mathrm{M}}$ ). In the last column of the bottom panel, we give the values of $\mathrm{SN}_{\mathrm{T}}$ obtained by summing the corresponding predicted values of $\mathrm{SN}_{\mathrm{N}}$ (top panel) and $\mathrm{SN}_{\mathrm{S}}$ (middle panel).

Predictions for the values of $\mathrm{SN}_{\mathrm{N}}$

$\begin{array}{lcc}\text { At the epoch } & \text { Predicted value } & \text { Used value of } \\ T_{\mathrm{M}} & 45 \pm 4.4 & \text { RNA } \\ T_{\mathrm{W}} & 48 \pm 4.4 & \text { WNA } \\ T_{\mathrm{N}} & 57 \pm 4.4 & \text { NAM } \\ T_{\mathrm{S}} & 43 \pm 4.4 & \text { SNA }\end{array}$

Predictions for the values of $\mathrm{SN}_{\mathrm{S}}$

$\begin{array}{lcc}\text { At the epoch } & \text { Predicted value } & \text { Used value of } \\ T_{\mathrm{M}} & 73 \pm 6.8 & \text { RSA } \\ T_{\mathrm{W}} & 78 \pm 6.8 & \text { WSA } \\ T_{\mathrm{N}} & 41 \pm 6.8 & \text { NSA } \\ T_{\mathrm{S}} & 79 \pm 6.8 & \text { SAM }\end{array}$

Predictions of for the values of $\mathrm{SN}_{\mathrm{T}}$

\begin{tabular}{lccc} 
At the epoch & Predicted value & Used value of & $\mathrm{SN}_{\mathrm{N}}+\mathrm{SN}_{\mathrm{S}}$ \\
$T_{\mathrm{M}}$ & $127 \pm 12$ & RWA & $\approx 118$ \\
$T_{\mathrm{W}}$ & $135 \pm 12$ & WAM & $\approx 126$ \\
$T_{\mathrm{N}}$ & $106 \pm 12$ & NWA & $\approx 98$ \\
$T_{\mathrm{S}}$ & $131 \pm 12$ & SWA & $\approx 122$ \\
$T_{\mathrm{M}}$ & $130 \pm 12$ & RWA $^{\mathrm{a}}$ & \\
\hline
\end{tabular}

these values of $R_{\mathrm{M}}$ of Sunspot Cycle 25 is slightly larger than the observed value of $R_{\mathrm{M}}$ of Sunspot Cycle 24. As already mentioned above the predicted values of RWA of Solar Cycle 25 are considerably smaller than the observed value of RWA of Solar Cycle 24. However, this comparison seems to be not genuine because the latter is an outlier (far from one-rms level) in the $A_{\mathrm{W}}-R_{\mathrm{M}}$ linear relationship shown in Figure 6 of Javaraiah (2021).

We determined the best-fit linear relation between the 13-month smoothed monthly mean values of sunspot-group area and SN. Figures 10, 11, and 12 show the relations between NSGA and $\mathrm{SN}_{\mathrm{N}}, \mathrm{SSGA}$ and $\mathrm{SN}_{\mathrm{S}}$, and WSGA and $\mathrm{SN}_{\mathrm{T}}$, where the subscripts N, S, and T indicate north, south, and total. All these linear relationships are statistically highly significant (the values of PROB are high). By using in these relations the values predicted above for RWA, RNA, RSA, WNA, WSA, NAM, and SAM we get/predict the values of $\mathrm{SN}_{\mathrm{N}}, \mathrm{SN}_{\mathrm{S}}$, and $\mathrm{SN}_{\mathrm{T}}$ (it is nothing but $R_{\mathrm{M}}$ at the epoch of $R_{\mathrm{M}} / \mathrm{RWA}$ ) of Sunspot Cycle 25 and 
the corresponding values at the maxima of 25th NSGA cycle, SSGA cycle, and WSGA cycle. The predicted values are also shown in Figures 10-12 and listed in Table 4, and in Figure 13 we compare these predicted values of Sunspot Cycle 25 with the corresponding observed values of Sunspot Cycle 24. The values shown in Figure 13 for $R_{\mathrm{M}}$ of Solar Cycle 25 are considerably larger than the value $(86 \pm 18)$ predicted earlier by Javaraiah (2021) by using the value predicted for RWA from a different method. As we can see in Figure 13 the values predicted here for $R_{\mathrm{M}}$ of Solar Cycle 25 also appear to be slightly larger than that of Solar Cycle 24 (the corresponding difference is negligible if we take the uncertainty into account). There is also a suggestion that the activity in the southern hemisphere is dominant in Solar Cycle 25, similarly to the case of sunspot-group area shown in Figure 9, except at the peak of NSGA, in all the remaining cases including the case of $R_{\mathrm{M}}$. The southern hemisphere's peak may coincide with whole sphere's peak. In fact, the overall pattern of the predicted values of Solar Cycle 25 closely resembles to that of Solar Cycle 24. Opposite to that seen in Figure 9 for the case of sunspot-group area, in Figure 13 there is a suggestion that on average Sunspot Cycle 25 would be slightly larger than Sunspot Cycle 24. The reason for this difference is not clear, but one possibility is that the ratio of small to large sunspots during the maximum of Solar Cycle 25 would be larger than that during the maximum of Solar Cycle 24. However, the differences between Solar Cycles 24 and 25 in sunspot-group area and also between Sunspot Cycles 24 and 25 are statistically insignificant. On the other hand properties of sunspot number and sunspots area cycles are not exactly the same. For example, the well-known Waldmeier effect of sunspot cycles is not present in the cycles of sunspot-group area (Dikpati, Gilman, and de Toma, 2008; Javaraiah, 2019). In several solar cycles, there exist some differences in the maximum epochs of sunspot-number and sunspot-area cycles and there are also differences in the relative heights of the peaks of some sunspot-number and sunspot-area cycles.

As already mentioned above, because the cosine best-fits of most of the parameters are not very accurate, although the area-SN linear relationships have small rms values it may be still difficult to draw a definite conclusion from these predicted values about the dominant hemisphere. On the other hand, uncertainty in the relative north-south asymmetry of any two quantities is much smaller than those of the corresponding absolute values (Javaraiah and Gokhale, 1997). Therefore we calculated the relative north-south asymmetry in RWA, WAM, NWA, and SWA and determined the cosine fit to the relative north-south asymmetry in each of these quantities. It would help, besides to find the long-term periodicity in north-south asymmetry of each quantity, to predict the north-south asymmetry in the corresponding quantities of future cycles. Figures $14 \mathrm{a}, 14 \mathrm{~b}, 14 \mathrm{c}$, and $14 \mathrm{~d}$ show the best-fit cosine curves of the relative north-south asymmetry $(\mathrm{RNA}-\mathrm{RSA}) /(\mathrm{RNA}+\mathrm{RSA})$ in RWA, $(\mathrm{WNA}-\mathrm{WSA}) /(\mathrm{WNA}+\mathrm{WSA})$ in WAM, $(\mathrm{NAM}-\mathrm{NSA}) /(\mathrm{NAM}+\mathrm{NSA})$ in NWA, and $(\mathrm{SNA}-\mathrm{SAM}) /(\mathrm{SNA}+\mathrm{SAM})$ in SWA, respectively. The best-fit cosine curves suggest the existence of $\approx 6$-cycle (66-year), $\approx 6$-cycle (66-year), $\approx 4.5$-cycle $(50$ year), and $\approx 5$.5-cycle (61-year) periodicities for the north-south asymmetry in RWA, WAM, NWA, and SWA, i.e. at the epochs of $R_{\mathrm{M}}$, WAM, NAM, and SAM, respectively. We have extrapolated the best-fit cosine curves and predicted the 

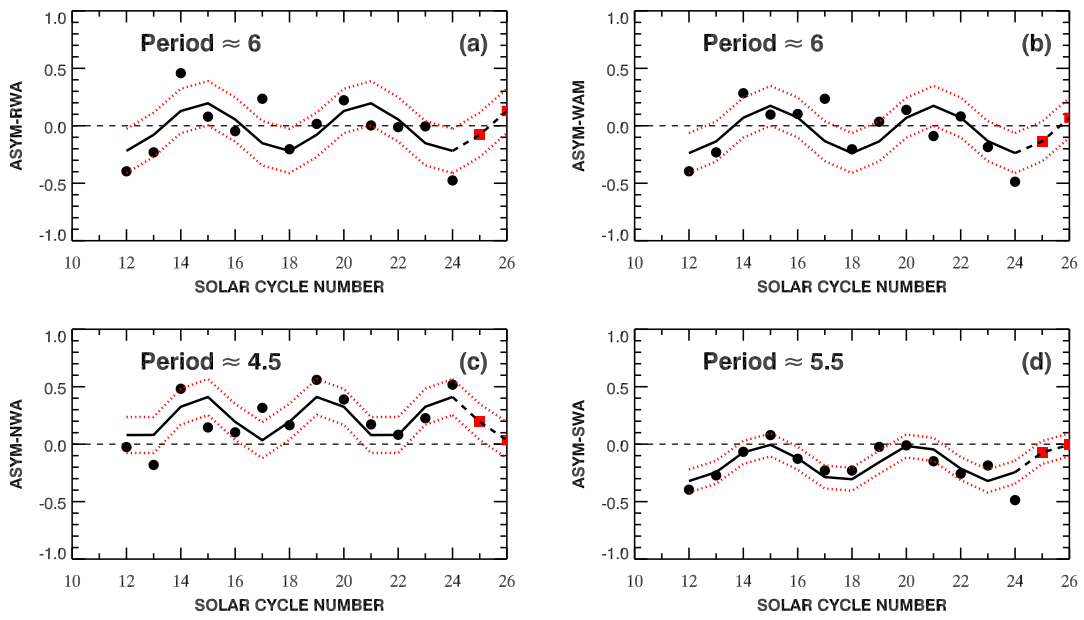

Figure 14. The continuous curve represents the best-fit cosine function to the values of the relative north-south asymmetry (a) in RWA, i.e. (RNA-RSA)/(RNA+RSA), (b) in WAM i.e. $(\mathrm{WNA}-\mathrm{WSA}) /(\mathrm{WNA}+\mathrm{WSA})$, (c) in NWA, i.e. (NAM-NSA)/(NAM+NSA), and (d) in SWA, i.e. (SNA-SAM)/(SNA+SAM). The dotted curve (red) represents the one-rms level. The period (in cycles) of the best-fit cosine function is also shown. The dashed portion of the curve is obtained by extrapolation and the filled squares (red) represent the predicted value of the north-south asymmetry in the corresponding parameters of Solar Cycles 25 and 26.

Figure 15: Open square (filled square) and open circle (filled circle) represent the values of northsouth asymmetry in the observed (predicted) sunspot-group area and SN shown in Figures 9 and 13, respectively, at $T_{\mathrm{M}}, T_{\mathrm{W}}, T_{\mathrm{N}}$, a spectively, at $T_{\mathrm{M}}, T_{\mathrm{W}}, T_{\mathrm{N}}$, and of the 24th (25th) SN, WSGA, NSGA, and SSGA cycles, respecNSGA, and SSGA cycles, respectively (no real-time differences are indicated along the $X$-axis). The filled triangle represents the values of the north-south asymmetry in the sunspot-group area at the maximum epochs of the 25th $\mathrm{SN}_{\mathrm{T}}$ WSGA, NSGA, and SSGA cycle obtained by extrapolating the bestfit cosine curves shown in Figure 14

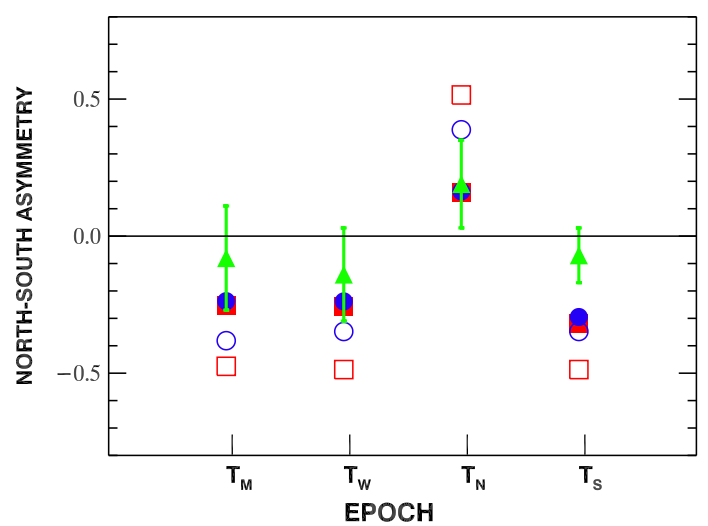

relative north-south asymmetry in these quantities at the maximum epochs of the 25th SN, WSGA, NSGA, and SSGA cycles. We find the values $-0.08 \pm 0.19$, $-0.14 \pm 0.17,0.19 \pm 0.16$, and $-0.07 \pm 1.0$ for the north-south asymmetry in RWA, WAM, NWA, and SWA of these cycles, respectively. Most of these values do not significantly differ from zero. Figure 15 shows, besides these values, the values of north-south asymmetry in sunspot group area and sunspot number shown in Figures 9 and 13, respectively, that is, in this figure essentially we compare the predicted values of the aforementioned 25 th cycle with the observed values of the corresponding 24th cycle. As we can see in this figure, the pattern of the predicted value of 25 th cycle is almost the same as that of the observed values of the 24 th cycle, that is there is a suggestion that except at the epoch of NAM, in 
all the remaining cases, including at the epoch of $R_{\mathrm{M}}$, the southern hemisphere is dominant during both Solar Cycles 24 and 25. There is a suggestion that overall the asymmetry in Solar Cycle 25 would be smaller than that of Solar Cycle 24.

\section{Discussion and Conclusions}

In most of the solar cycles, activity in the northern and southern hemispheres peaks at different times, that is at least one of the hemispheric maxima frequently does not coincide with the whole sphere (total) maximum. Typically, one hemisphere peaks well before the other, and the maximum of the whole-Sun area or sunspot number can be the peak in one hemisphere, or the peak in the other hemisphere, or something in between. Predictions for each hemisphere's maximum and the corresponding north-south asymmetry of a solar cycle may help to understand the mechanisms of the solar cycle, the solar-terrestrial relationship, and solar-activity influence on space weather. Here we analysed the GPR and DPD sunspot-group data during 1874-2017 and studied the cycleto-cycle variations in the values of WSGA, NSGA, and SSGA at the epochs of the maxima of Sunspot (SN) Cycles $12-24$ and at the epochs of the maxima of the 12th to 24th WSGA, NSGA, and SSGA cycles. We find that, except in the cases of SSGA at the peak of WSGA and NSGA at the peak of SSGA, in all the remaining cases the $\mathrm{G}-\mathrm{O}$ rule is violated by the solar cycle pair $(22,23)$. Using the G-O rule, we obtained a value of SSGA at the maximum of WSGA Cycle 25 that is much larger than the value of SSGA at the maximum of WSGA Cycle 24. Similarly, we obtained a value of NSGA at the maximum of SSGA Cycle 25 that is also found to be larger than the value of NSGA at the maximum of SSGA Cycle 24. However, these predictions are somewhat unreliable because the $\mathrm{G}-\mathrm{O}$ rule cannot be used to predict the amplitude of an odd-numbered solar cycle without prior knowledge about non-violation of this rule by the corresponding pair of even- and odd-numbered solar cycles. We determined the best-fit cosine functions to the values of each of the parameters given in Table 1 . We find that there exists a $\approx 132$-year periodicity in all the northern hemisphere's parameters and the 54-66-year periodicity in all the southern hemisphere's parameters. By extrapolating the best-fit cosine curves we make cautious predictions for the values of the parameters of the corresponding 25th cycles. The sums of the predicted values of the parameters of northern and southern hemispheres represent the predictions for the corresponding value of the whole sphere. It is found that the amplitudes of the 25th WSGA and SSGA cycles would be to some extent smaller than those of the corresponding 24th cycle. The amplitude of 25th NSGA cycle would be almost the same as that of the corresponding 24 th cycle. The value of WSGA at the maximum epoch of Sunspot Cycle 25 would also be smaller than that at the maximum epoch of Sunspot Cycle 24, i.e. the overall Solar Cycle 25 in sunspot-group area would be smaller than Solar Cycle 24 in sunspot-group area. We obtained a good linear relationship between the 13-month smoothed monthly mean SN and sunspot-group area in the whole sphere and also in the northern and the southern hemispheres. By inputting the predicted values of RWA, RNA, and RSA in these relationships we obtained the 
values of SN at the epochs of maxima of the 25th SN, WSGA, NSGA, and SSGA cycles. The obtained value $(130 \pm 12)$ of $R_{\mathrm{M}}$ of Sunspot Cycle 25 is found to be slightly larger than that of Sunspot Cycle 24. Except at the maximum of NSGA Cycle 25 where the north is found to be dominant in strength of activity, the values of SN at the maxima of WSGA Cycle 25, SSGA Cycle 25, and Sunspot Cycle 25 the south is found to be dominant. The cosine best-fits to the values of the corresponding north-south asymmetry in WSGA, NSGA, and SSGA at the maxima of the 12th to 24th SN, WSGA, NSGA, and SSGA cycles suggest the existence of 50-66-year periodicities in the north-south asymmetry and the same aforementioned pattern of hemispheric dominance. Overall, the present analysis suggests that the amplitude of Solar Cycle 25 would be slightly larger than that of Solar Cycle 24 and the activity in the southern hemisphere would be dominant.

All the parameters of the solar cycles of a hemisphere have the same periodicity, implying that different parameters of the hemisphere are at different phases of the same long-period cycle; that is, the epochs of different parameters of the northern hemisphere represent different phases of the same cosine wave of $\approx 132$-year period and the different parameters of the southern hemisphere represent different phases of the same cosine wave of 54-66-year period. The large difference in the periods of the cosine waves of northern and southern hemispheres' activity may be responsible for varying phase differences between 11-year cycles of northern and southern hemispheres' activity. It may be worth finding why there exists a large difference in the long-term periodicities in northern and southern hemispheres' activity (which is beyond the scope of the present analysis).

The best-fit cosine functions of sunspot-number data have already indicated that Solar Cycle 25 is a reasonably small cycle and constitutes the minimum of the upcoming new Gleissberg cycle (Javaraiah, Bertello, and Ulrich, 2005; Javaraiah, 2017). A number of studies on long-term variations in solar activity by using different techniques have also predicted Dalton-minimum-like characteristics around Solar Cycle 25 (Javaraiah, 2015; Gao, 2016; Coban et al., 2021). In the present analysis, we also find the same. However, whether Solar Cycle 25 will be smaller or larger than the reasonably small Solar Cycle 24 is yet to be established. Many authors predicted the amplitude of Solar Cycle 25 by using different techniques (for recent reviews see Petrovay, 2020; Nandy, 2021). Some authors predicted a small amplitude, some others predicted a large amplitude for Solar Cycle 25, and a larger number of authors predicted that it would be approximately the same size as Solar Cycle 24.

It is widely believed that the strength of polar fields at the minimum of an upcoming solar cycle is a good physically oriented precursor to predict the strength of the solar cycle. From this method and in flux-transport dynamo models it is predicted that Solar Cycle 25 would have the same size or slightly larger than that of Solar Cycle 24 (Nandy, 2021; Kumar et al., 2021) and our prediction here closely agrees with it. As already mentioned earlier we found a specific method (Javaraiah, 2007), and using it we predicted a small value for the amplitude of Solar Cycle 25, strongly implying that Solar Cycle 25 will be considerably smaller than Solar Cycle 24 (Javaraiah, 2015; 2017; 2021). However, the predictions made 
in the present analysis by using a basic method suggest that Solar Cycle 25 in sunspot-group area would be slightly smaller than Solar Cycle 24 in sunspotgroup area, but Sunspot Cycle 25 would be slightly larger than Sunspot Cycle 24 . A reason for this discrepancy in our present and earlier predictions for Sunspot Cycle 25 is not known to us. There are some inconsistencies in both earlier and present analyses. Regarding the earlier method, in the case of Solar Cycle 24 its second SN peak (coinciding with the strong southern hemisphere's peak, see Figure 1) is taken into account because it is substantially larger than its first peak (coinciding with the weak northern hemisphere peak), whereas in the case of most of the previous cycles the respective first peak is taken into account because it is larger than the corresponding second peak. In addition, since the uncertainties in the measured areas of sunspot groups are not known, in the earlier analyses the linear least-squares fit calculations were not included the uncertainties in the area-sums (sums of the areas of sunspot groups; see Section 1). Here we repeated the earlier calculations by taking $10 \%$ of the area-sums for their uncertainties (note that $R_{\mathrm{M}}$ values have $5 \%-10 \%$ uncertainties). None of the best-fit linear relations, namely between the area-sum and $R_{\mathrm{M}}$ and areasum and RWA, etc. are found statistically significant (PROB values are found considerably small). However, the $10 \%$ uncertainties in the area-sums seem to be underestimated. To get a reasonably large PROB it seems necessary to use $20-$ $25 \%$ of the area-sums for their uncertainties, which may be too unrealistic. In the present analysis the results of best-fit cosine functions are suggestive rather than compelling (best-fit cosine functions have to some extent large $\chi^{2}$-values). The predictions made from our earlier method are based on the existence of a strong connection (high correlation) between consecutive cycles. The values predicted here from the long-term cyclic trends in the data might be to some extent overestimated. Further investigations are needed to conclude whether our present or earlier predictions will be right or wrong. On the other hand the value predicted in the present analysis for the amplitude of Solar Cycle 25 is only slightly larger than that of the reasonable small Solar Cycle 24 and considerably smaller than the average amplitude 178.7 of solar cycles (Pesnell, 2018). Hence in the present analysis we confirmed that the beginning of the upcoming Gleissberg cycle would take place around Solar Cycle 25 (see the cosine curve in Figure 7).

Our present predictions (see Table 4) for Solar Cycle 25 are somewhat comparable to the following predictions made by different authors using different techniques. Rigozo et al. (2011) by extrapolating the sunspot-number timeseries spectral components estimated a maximum sunspot number of 132.1 for Solar Cycle 25. Cameron, Jiang, and Schüssler (2016) by using a flux-transport dynamo model simulated a value of dipole moment around 2020, i.e. around the beginning of Solar Cycle 25, and suggested that Solar Cycle 25 will be of moderate amplitude, not much higher than that of Solar Cycle 24. Pesnell and Schatten (2018) applied a precursor method by using the SODA Index, which is determined by polar magnetic fields and the spectral index, predicted a large amplitude $(135 \pm 25)$ for Solar Cycle 25. Jiang et al. (2018) used a solar surface flux-transport model and predicted that the peak sunspot number of Solar Cycle 25 is $125 \pm 32$. Petrovay et al. (2018) using coronal green-line data as a precursor predicted 130 for the amplitude of Solar Cycle 25. Bhowmik and 
Nandy (2018) using magnetic-field evolution models for the Sun's surface and interior predicted that Solar Cycle 25 will be similar or slightly stronger than Solar Cycle 24. Du (2020) using the rate of decrease in the smoothed monthly mean sunspot number over the final three years of Solar Cycle 24 predicted $130.0 \pm 31.9$ for the amplitude of Solar Cycle 25. Kumar et al. (2021) used the polar-precursor method and predicted $126 \pm 3$ for the amplitude of Solar Cycle 25. Ahluwalia (2022) using data for sunspot numbers and geomagnetic indices (aa/Ap) and their heuristic methodology inferred that Solar Cycle 25 may be as active as Solar Cycle 24 ruling out a Dalton-like minimum in the 21st century. Upton and Hathaway (2018) using an advective flux transport code found evidence that during Solar Cycle 25 the southern hemisphere will be more active than the northern hemisphere. Gopalswamy et al. (2018) used a precursor method (microwave-imaging observations) and predicted that the sunspot-number peaks of southern and northern hemispheres during Sunspot Cycle 25 will be 89 and 59, respectively. These indicate a considerable northsouth asymmetry corresponding to the amplitude of Solar Cycle 25 and that activity would be dominant in the southern hemisphere. Werner and Guineva (2020) using auto-regressive models of different order predicted 117 for the amplitude of Solar Cycle 25 and that solar activity would be dominant in the southern hemisphere. Pishkalo (2021) using the absolute values of the mean polar magnetic fields during the two-year interval just before the cycle minimum in northern and southern hemispheres as precursors predicted $66 \pm 17$ and $83 \pm 21$ for northern and southern hemispheres' amplitudes of Solar Cycle 25. Labonville, Charbonneau, and Lemerle (2019) from a data-driven solar cycle model predicted a low amplitude for Solar Cycle 25 and the northern hemisphere about $20 \%$ more than the south southern hemisphere. Our earlier analysis (Javaraiah, 2021) also suggested that at the epoch of maximum of the Solar Cycle 25 the activity will be about $20 \%$ more in northern hemisphere than that in southern hemisphere, whereas our present analysis suggests that the southern hemisphere would be dominant. According to the international panel to forecast Solar Cycle 25, the amplitude of this cycle would be 115, which is almost the same as the amplitude of Solar Cycle 24 (see www.swpc.noaa.gov/news).

Acknowledgments The author thanks the anonymous reviewer for useful comments and suggestions. The author acknowledges the work of all the people who contribute to and maintain the GPR and DPD Sunspot databases. The sunspot-number data are provided by WDC-SILSO, Royal Observatory of Belgium, Brussels.

\section{Declarations}

Conflict of interest The author declares that he has no conflicts of interest.

Data Availability All data generated or analysed during this study are included in this article.

\section{References}

Ahluwalia, H.S.: 2022, Forecast for sunspot cycle 25 activity. Adv. Space Res. 69, 794. DOI. ADS. 
Baranyi, T., Győri, L., Ludmány, A.: 2016, On-line Tools for Solar Data Compiled at the Debrecen Observatory and Their Extensions with the Greenwich Sunspot Data. Solar Phys. 291, 3081. DOI. ADS.

Bhowmik, P., Nandy, D.: 2018, Prediction of the strength and timing of sunspot cycle 25 reveal decadal-scale space environmental conditions. Nat. Commun. 9, 5209. DOI. ADS.

Cameron, R.H., Jiang, J., Schüssler, M.: 2016, Solar Cycle 25: Another Moderate Cycle? Astrophys. J. Lett. 823, L22. DOI. ADS.

Carbonell, M., Oliver, R., Ballester, J.L.: 1993, On the asymmetry of solar activity. Astron. Astrophys. 274, 497. ADS.

Chowdhury, P., Gokhale, M.H., Singh, J., Moon, Y.-J.: 2016, Mid-term quasi-periodicities in the CaII-K plage index of the Sun and their implications. Astrophys. Space Sci. 361, 54. DOI. ADS.

Coban, G.C., Raheem, A.-u., Cavus, H., Asghari-Targhi, M.: 2021, Can Solar Cycle 25 Be a New Dalton Minimum? Solar Phys. 296, 156. DOI. ADS.

Deng, L.H., Xiang, Y.Y., Qu, Z.N., An, J.M.: 2016, Systematic Regularity of Hemispheric Sunspot Areas Over the Past 140 Years. Astron. J. 151, 70. DOI. ADS.

Dikpati, M., Gilman, P.A., de Toma, G.: 2008, The Waldmeier Effect: An Artifact of the Definition of Wolf Sunspot Number? Astrophys. J. Lett. 673, L99. DOI. ADS.

Du, Z.: 2020, Predicting the Amplitude of Solar Cycle 25 Using the Value 39 Months Before the Solar Minimum. Solar Phys. 295, 147. DOI. ADS.

Gao, P.X.: 2016, Long-term Trend of Sunspot Numbers. Astrophys. J. 830, 140. DOI. ADS.

Gnevyshev, M.N., Ohl, A.I.: 1948, On the 22-year cycle of solar activity. Astron. Zh. 25, 18.

Gopalswamy, N., Mäkelä, P., Yashiro, S., Akiyama, S.: 2018, Long-term solar activity studies using microwave imaging observations and prediction for cycle 25. J. Atmos. Solar-Terr. Phys. 176, 26. DOI. ADS.

Győri, L., Baranyi, T., Ludmány, A.: 2011, Photospheric data programs at the Debrecen Observatory. In: Prasad Choudhary, D., Strassmeier, K.G. (eds.) Physics of Sun and Star Spots, IAU Symp. 273, Cambridge Univ. Press, Cambridge, UK, 403. DOI. ADS.

Győri, L., Ludmány, A., Baranyi, T.: 2017, Comparative analysis of Debrecen sunspot catalogues. Mon. Not. Roy. Astron. Soc. 465, 1259. DOI. ADS.

Hathaway, D.H.: 2015, The Solar Cycle. Liv. Rev. Solar Phys. 12, 4. DOI. ADS.

Javaraiah, J.: 2007, North-south asymmetry in solar activity: predicting the amplitude of the next solar cycle. Mon. Not. Roy. Astron. Soc. 377, L34. DOI. ADS.

Javaraiah, J.: 2008, Predicting the Amplitude of a Solar Cycle Using the North - South Asymmetry in the Previous Cycle: II. An Improved Prediction for Solar Cycle 24. Solar Phys. 252, 419. DOI. ADS.

Javaraiah, J.: 2015, Long-term variations in the north-south asymmetry of solar activity and solar cycle prediction, III: Prediction for the amplitude of solar cycle 25. New Astron. 34, 54. DOI. ADS.

Javaraiah, J.: 2017, Will Solar Cycles 25 and 26 Be Weaker than Cycle 24? Solar Phys. 292, 172. DOI. ADS.

Javaraiah, J.: 2019, North-South Asymmetry in Solar Activity and Solar Cycle Prediction, IV: Prediction for Lengths of Upcoming Solar Cycles. Solar Phys. 294, 64. DOI. ADS.

Javaraiah, J.: 2020, Long-term Periodicities in North-south Asymmetry of Solar Activity and Alignments of the Giant Planets. Solar Phys. 295, 8. DOI. ADS.

Javaraiah, J.: 2021, North-south asymmetry in solar activity and Solar Cycle prediction, V: prediction for the north-south asymmetry in the amplitude of Solar Cycle 25. Astrophys. Space Sci. 366, 16. DOI. ADS.

Javaraiah, J., Gokhale, M.H.: 1997, Periodicities in the North-South Asymmetry of the Solar Differential Rotation and Surface Magnetic Field. Solar Phys. 170, 389. DOI. ADS.

Javaraiah, J., Bertello, L., Ulrich, R.K.: 2005, Long-Term Variations in Solar Differential Rotation and Sunspot Activity. Solar Phys. 232, 25. DOI. ADS.

Jiang, J., Wang, J.-X., Jiao, Q.-R., Cao, J.-B.: 2018, Predictability of the Solar Cycle Over One Cycle. Astrophys. J. 863, 159. DOI. ADS.

Knaack, R., Stenflo, J.O., Berdyugina, S.V.: 2004, Periodic oscillations in the north-south asymmetry of the solar magnetic field. Astron. Astrophys. 418, L17. DOI. ADS.

Kumar, P., Nagy, M., Lemerle, A., Karak, B.B., Petrovay, K.: 2021, The Polar Precursor Method for Solar Cycle Prediction: Comparison of Predictors and Their Temporal Range. Astrophys. J. 909, 87. DOI. ADS.

Labonville, F., Charbonneau, P., Lemerle, A.: 2019, A dynamo-based forecast of solar cycle. Solar Phys. 294, 82. DOI. ADS. 
Li, K.J., Wang, J.X., Xiong, S.Y., Liang, H.F., Yun, H.S., Gu, X.M.: 2002, Regularity of the north-south asymmetry of solar activity. Astron. Astrophys. 383, 648. DOI. ADS.

Mandal, S., Banerjee, D.: 2016, Sunspot Sizes and the Solar Cycle: Analysis Using Kodaikanal White-light Digitized Data. Astrophys. J. Lett. 830, L33. DOI. ADS.

McIntosh, S.W., Leamon, R.J., Gurman, J.B., Olive, J.-P., Cirtain, J.W., Hathaway, D.H., Burkepile, J., Miesch, M., Markel, R.S., Sitongia, L.: 2013, Hemispheric Asymmetries of Solar Photospheric Magnetism: Radiative, Particulate, and Heliospheric Impacts. Astrophys. J. 765, 146. DOI. ADS.

Muraközy, J., Ludmány, A.: 2012, Phase lags of solar hemispheric cycles. Mon. Not. Roy. Astron. Soc. 419, 3624. DOI. ADS.

Nagovitsyn, Y.A., Osipova, A.A., Nagovitsyna, E.Y.: 2021, "Generative" Indices of Sunspot Solar Activity: 145-Year Composite Series. Solar Phys. 296, 32. DOI. ADS.

Nandy, D.: 2021, Progress in Solar Cycle Predictions: Sunspot Cycles 24-25 in Perspective. Solar Phys. 296, 54. DOI. ADS.

Nepomnyashchikh, A., Mandal, S., Banerjee, D., Kitchatinov, L.: 2019, Can the long-term hemispheric asymmetry of solar activity result from fluctuations in dynamo parameters? Astron. Astrophys. 625, A37. DOI. ADS.

Norton, A.A., Gallagher, J.C.: 2010, Solar-Cycle Characteristics Examined in Separate Hemispheres: Phase, Gnevyshev Gap, and Length of Minimum. Solar Phys. 261, 193. DOI. ADS.

Norton, A.A., Charbonneau, P., Passos, D.: 2014, Hemispheric Coupling: Comparing Dynamo Simulations and Observations. Space Sci. Rev. 186, 251. DOI. ADS.

Pesnell, W.D.: 2018, Effects of Version 2 of the International Sunspot Number on Naïve Predictions of Solar Cycle 25. Space Weather 16, 1997. DOI. ADS.

Pesnell, W.D., Schatten, K.H.: 2018, An Early Prediction of the Amplitude of Solar Cycle 25. Solar Phys. 293, 112. DOI. ADS.

Petrovay, K.: 2020, Solar cycle prediction. Liv. Rev. Solar Phys. 17, 2. DOI. ADS.

Petrovay, K., Nagy, M., Gerják, T., Juhász, L.: 2018, Precursors of an upcoming solar cycle at high latitudes from coronal green line data. J. Atmos. Solar-Terr. Phys. 176, 15. DOI. ADS.

Pishkalo, M.I.: 2021, Prediction of Solar Cycle 25: Maximum in the N- and S-Hemispheres. Kinemat. Phys. Celest. Bodies 37, 27. DOI. ADS.

Ravindra, B., Javaraiah, J.: 2015, Hemispheric asymmetry of sunspot area in solar cycle 23 and rising phase of solar cycle 24: Comparison of three data sets. New Astron. 39, 55. DOI. ADS.

Ravindra, B., Chowdhury, P., Javaraiah, J.: 2021, Solar-Cycle Characteristics in Kodaikanal Sunspot Area: North-South Asymmetry, Phase Distribution and Gnevyshev Gap. Solar Phys. 296, 2. DOI. ADS.

Rigozo, N.R., Souza Echer, M.P., Evangelista, H., Nordemann, D.J.R., Echer, E.: 2011, Prediction of sunspot number amplitude and solar cycle length for cycles 24 and 25. J. Atmos. Solar-Terr. Phys. 73, 1294. DOI. ADS.

Schüssler, M., Cameron, R.H.: 2018, Origin of the hemispheric asymmetry of solar activity. Astron. Astrophys. 618, A89. DOI. ADS.

Upton, L.A., Hathaway, D.H.: 2018, An Updated Solar Cycle 25 Prediction With AFT: The Modern Minimum. Geophys. Res. Lett. 45, 8091. DOI. ADS.

Verma, V.K.: 1993, On the North-South Asymmetry of Solar Activity Cycles. Astrophys. J. 403, 797. DOI. ADS.

Werner, R., Guineva, V.: 2020, Forecasting sunspot numbers for solar cycle 25 using autoregressive models for both hemispheres of the sun. Comptes rendus de 1'Acadmíe bulgare des Sciences 73, 82. DOI.

Zolotova, N.V., Ponyavin, D.I., Arlt, R., Tuominen, I.: 2010, Secular variation of hemispheric phase differences in the solar cycle. Astron. Nach. 331, 765. DOI. ADS. 